University of Nebraska - Lincoln

DigitalCommons@University of Nebraska - Lincoln

Faculty Publications: Materials Research

Science and Engineering Center

Materials Research Science and Engineering

Center

2006

\title{
Surface Segregation in Multicomponent Clusters
}

Peter A. Dowben

University of Nebraska-Lincoln, pdowben@unl.edu

Ning Wu

University of Nebraska-Lincoln

Natalie Palina

Physikalisches Institut der Universität Bonn, Nussallee 12, D-53115 Bonn, Germany, palina@physik.unibonn.de

H. Modrow

Physikalisches Institut der Universität Bonn, Nussallee 12, D-53115 Bonn, Germany

R. Müller

Institut für Physikalische Hochtechnologie, Albert-Einstein-Straße 9, D-07745 Jena, Germany

See next page for additional authors

Follow this and additional works at: https://digitalcommons.unl.edu/mrsecfacpubs

Part of the Materials Science and Engineering Commons

Dowben, Peter A.; Wu, Ning; Palina, Natalie; Modrow, H.; Müller, R.; Hormes, J.; and Losovyj, Yaroslav B., "Surface Segregation in Multicomponent Clusters" (2006). Faculty Publications: Materials Research Science and Engineering Center. 82.

https://digitalcommons.unl.edu/mrsecfacpubs/82

This Article is brought to you for free and open access by the Materials Research Science and Engineering Center at DigitalCommons@University of Nebraska - Lincoln. It has been accepted for inclusion in Faculty Publications:

Materials Research Science and Engineering Center by an authorized administrator of DigitalCommons@University of Nebraska - Lincoln. 


\section{Authors}

Peter A. Dowben, Ning Wu, Natalie Palina, H. Modrow, R. Müller, J. Hormes, and Yaroslav B. Losovyj 


\section{Surface Segregation in Multicomponent Clusters}

Peter A. Dowben ${ }^{1}$, Ning Wu ${ }^{1}$, Natalie Palina ${ }^{2}$, H. Modrow ${ }^{2}$, R. Müller ${ }^{3}$, J. Hormes ${ }^{4}$, Ya.B. Losovyj $^{1,4}$

${ }^{1}$ Department of Physics and Astronomy and the Center for Materials Research and Analysis, University of Nebraska - Lincoln, Lincoln, NE 68588-0111, pdowben@ unl.edu

${ }^{2}$ Physikalisches Institut der Universität Bonn, Nussallee 12, D-53115 Bonn, Germany

${ }^{3}$ Institut für Physikalische Hochtechnologie, Albert-Einstein-Straße 9, D-07745 Jena, Germany

${ }^{4}$ Center for Advanced Microstructures and Devices, Louisiana State University, 6980

Jefferson Hwy, Baton Rouge, LA 70806, ylosovyj@1su.edu

\section{ABSTRACT}

Nanostructured materials are not immune from surface segregation, as can be shown for solid samples made from nanosized $\mathrm{BaFe}_{12-2 \mathrm{x}} \mathrm{Co}_{\mathrm{x}} \mathrm{Ti}_{\mathrm{x}} \mathrm{O}_{19}$ barium ferrite particles and a variety of free clusters. Both theory and experiment provide ample demonstration that very limited dimensions of very small clusters does not necessarily impart stability against surface and grain boundary segregation. In fact, with the larger surface to volume ratio in small clusters and lower average atomic coordination, we anticipate that compositional instabilities in small clusters will readily occur.

\section{INTRODUCTION}

Surface segregation is a long standing problem in materials science with great technological significance. The phenomenon of surface segregation is the preferential enrichment of one component of a multi-component system at a boundary or interface. Atomic size and lattice strain, bond strengths, and even magnetic ordering influence the extent of segregation. Surface segregation indicates that the surface enthalpy is different from the bulk and occurs at finite temperatures (or in the materials growth process) when barriers to diffusion are overcome. The difference in the total free energy of the surface with respect to the bulk is a consequence of the surface truncation to vacuum and the resultant breaking of symmetry.

Besides surface segregation, the free energy difference drives other phenomena in order to minimize the total free energy [1], such as surface relaxations and surface reconstructions. All these phenomena are related to changes in crystalline order in the surface, resulting in the creation of different electronic and magnetic properties at the surface with respect to the bulk. These different surface electronic structure signatures can be sometimes exploited to study surface segregation.

It is well known that surface segregation plays a crucial role in affecting the surface and interface polarization ferromagnets [2]. This is considered to be very important to many spintronic applications as polarization is strongly influenced by composition [3-8]. Evidence that surface composition affects spin polarization of 
potentially high polarization materials abounds $[3,4,6,9-13]$. One of the more studied examples of reduced spin-polarization due to surface compositional instabilities of the $\mathrm{NiMnSb}$ half-Heusler alloy [2,3,14]. Magnetism plays a role in other ways: segregation can alter the magnetic ordering at the surface and thus also alter the surface free energy.

Clusters pose a more extreme example of systems with large surface to volume ratios, and are thus far more susceptible to surface segregation. With different stable faces at a cluster surface, the segregation will not be uniform across the cluster surface.

Unfortunately, the surface composition of an isolated cluster surface is difficult to characterize, and supported clusters are difficult to examine without careful consideration of a number of substrate contributions. These complexities aside, we can show that van der Waals bonded clusters $[15,16]$ and even strongly ordered ferromagnetic alloy metal clusters will exhibit considerable surface segregation. As a result, for cluster assembled materials one cannot assume that because of the small cluster size there must exist compositional uniformity. The opposite is true: the smaller the cluster size in cluster assembled materials, the more likely the resulting material resembles ensemble of different compositional materials.

\section{CHARACTERIZING SURFACE SEGREGATION}

The surface energy depends upon crystal orientation, the extent of crystallinity, defect and grain boundary concentrations, the temperature, surface reconstructions, and surface lattice relaxations. Surface segregation, surface reconstructions and lattice defects will all result in changes in the total energy, but are limited by very different kinetics.

It is now fairly well established that equilibrium segregation can extend beyond the "topmost" or outer most atomic layer to a few atomic layers away from the surface (into the bulk material). The characterization of surface segregation must therefore be not only surface sensitive, but composition to a depth of the order of a few atomic layers becomes essential. At present, these characterization requirements are met to a large extent by analysis techniques based on electron and ion spectroscopies [17-18]. These are Auger electron spectroscopy (AES) [19], angle-resolved X-ray photoelectron spectroscopy or angle-resolved ESCA i.e. electron spectroscopy for chemical analysis (ARXPS) [1,6,9-14,19], ion scattering spectroscopy (ISS) [20] and secondary ion mass spectrometry (SIMS) [21]. We have tended to focus on ARXPS as the technique used for characterizing the surface composition, as the technique is not "destructive", and among the most established methods to accurately assess surface composition. The technique does require modification if applied to the study of clusters.

In ARXPS surface composition can be determined with considerable accuracy since the effective probing depth becomes shorter as the emission angle is increased with respect to the surface normal. The experimental core level intensities for any two components from a multi-component alloy are acquired at several emission angles $\theta$, usually from $\theta=0^{\circ}$ (normal emission) to $60^{\circ}$ (off-normal emission). Then, a linear background contribution is systematically subtracted from each raw spectrum. The peak intensities are further normalized by the corresponding differential cross-section for emission and by the analyzer transmission function [1,9-12,22]. The experimentally normalized intensity ratio for any two elements $\mathrm{A}$ and $\mathrm{B}$ is thus given: 


$$
R^{\exp }(\theta)=\frac{I(A) / \sigma_{A}}{I(B) / \sigma_{B}} \frac{T\left(E_{A}\right)}{T\left(E_{B}\right)}
$$

where $I(A)$ and $I(B)$ are the measured core level intensities for elements $A$ and $B, \sigma_{\mathrm{A}}$ and $\sigma_{\mathrm{B}}$ are the cross sections, and $T\left(E_{A}\right)$ and $T\left(E_{B}\right)$ are the transmission functions of the electron energy analyzer for elements $A$ and $B$ as a function of the corresponding photoelectron kinetic energies $E_{A}$ and $E_{B}$. The measured transmission functions must be determined for each analyzer. (For example, the transmission function of PHI 10-360 Precision Energy Analyzer is $T\left(E_{A}\right)=\left(E_{A}\right)^{1 / 2}[23,24]$. For the Gamma Scienta SES-100 the corresponding transmission functions have been determined to a little more complex [22]).

We can apply a simplified model to fit the experimental intensity ratios obtained from ARXPS. The comparison between theory and experiment is accomplished through considerations of the theoretical normalized intensity ratio of element $A$ and $B$ as given by:

$$
R^{\text {theor }}(\theta)=\frac{\sum_{j=0}^{\infty} f_{j}(A) \exp \left[\frac{-j d}{\lambda_{A}^{j} \cos (\theta)}\right]}{\sum_{j=0}^{\infty} f_{j}(B) \exp \left[\frac{-j d}{\lambda_{B}^{j} \cos (\theta)}\right]}
$$

where $\lambda_{A}^{j}$ and $\lambda_{B}^{j}$ are the inelastic mean free paths of the core electrons generated from elements $A$ and $B$ respectively and passing through the material contained in layer $j$. The inelastic mean free paths can be adopted from previously published methodologies [2526]. The atomic fraction of element $A$ (chosen as the element which segregates to the surface) in the $j^{\text {th }}$ layer below the surface is roughly given by:

$$
f_{j}(A)=b+\delta \exp (-j d / G)
$$

where $b$ is the bulk fraction of element $A, \delta$ and $G$ are fitting parameters representing the extent of the segregation and the segregation depth respectively, and $d$ is the distance between atomic layers. These two quantities are also the fitting parameters when comparing the model with experimental values. From the profile form $f_{j}(\mathrm{~A})$ one can calculate the apparent surface concentration (or relative intensity) of element $A$ for a particular core level. For strongly ordered alloys, different profile forms must be employed, and consideration of the likely short range order parameters cannot be neglected. Phase separation near the surface can further complicate analysis.

These complications are highlighted in some multicomponent oxides, in particular the manganese perovskites [10-13]. In the case of $\mathrm{La}_{0.65} \mathrm{~Pb}_{0.35} \mathrm{MnO}_{3}$, with a gentle annealing procedure (up to $250{ }^{\circ} \mathrm{C}$ ), the surface is dominated by appreciable $\mathrm{Pb}$ segregation, whereas a heavily annealed surface (up to $520{ }^{\circ} \mathrm{C}$ ) undergoes an irreversible restructuring into a Ruddlesden-Popper phase $\left(\mathrm{La}_{1-\mathrm{x}} \mathrm{Pb}_{\mathrm{x}}\right)_{2} \mathrm{MnO}_{4}$ [13], as indicated in Figure 1. For $\mathrm{La}_{1-\mathrm{x}} \mathrm{Ca}_{\mathrm{x}} \mathrm{MnO}_{3}(\mathrm{x}=0.1$ and 0.35$)$, the terminal layer is predominately $\mathrm{Mn}-\mathrm{O}$ for $\mathrm{x}=0.35$, while for $\mathrm{x}=0.1$, the majority of the surface is $\mathrm{La} / \mathrm{Ca}-\mathrm{O}$ terminated according to 
analysis of surface composition with XPS [10]. Sr surface segregation has also been found in $\mathrm{La}_{0.65} \mathrm{Sr}_{0.35} \mathrm{MnO}_{3}$, which causes a major restructuring of the surface region characterized by the formation of a Ruddlesden-Popper phase $(\mathrm{La}, \mathrm{Sr})_{n+1} \mathrm{Mn}_{n} \mathrm{O} 3_{n+1}$ with $n=1[11,12]$.

For clusters, these techniques have to be modified. Angle resolved techniques have to be replaced by comparing different core levels, with different photoelectron kinetic energies are different effective mean free paths, or by comparing select photoemission states at different photon energies. The equations above can be modified to extract a composition profile, as has been done for segregation studies of $\mathrm{Fe}-\mathrm{Cr}$ alloys [27-28], utilizing the different mean free paths of the signature photoelectron signals. At low kinetic energies, the mean free path should strongly scale as the inverse square of the freeelectron plasmon energy $\left(\propto \mathrm{E}_{\mathrm{p}}{ }^{-2}\right)$ or, alternatively, roughly proportional to the inverse of the number of valence electrons [29-31] but only if we disregard the spin dependent plasmon density and the metallicity (insulators typically have far longer mean free paths for electrons than metals). The spin-polarized electron mean free path, however, has been ascribed to the number $d$ band holes [32] and electron-electron scattering [33].
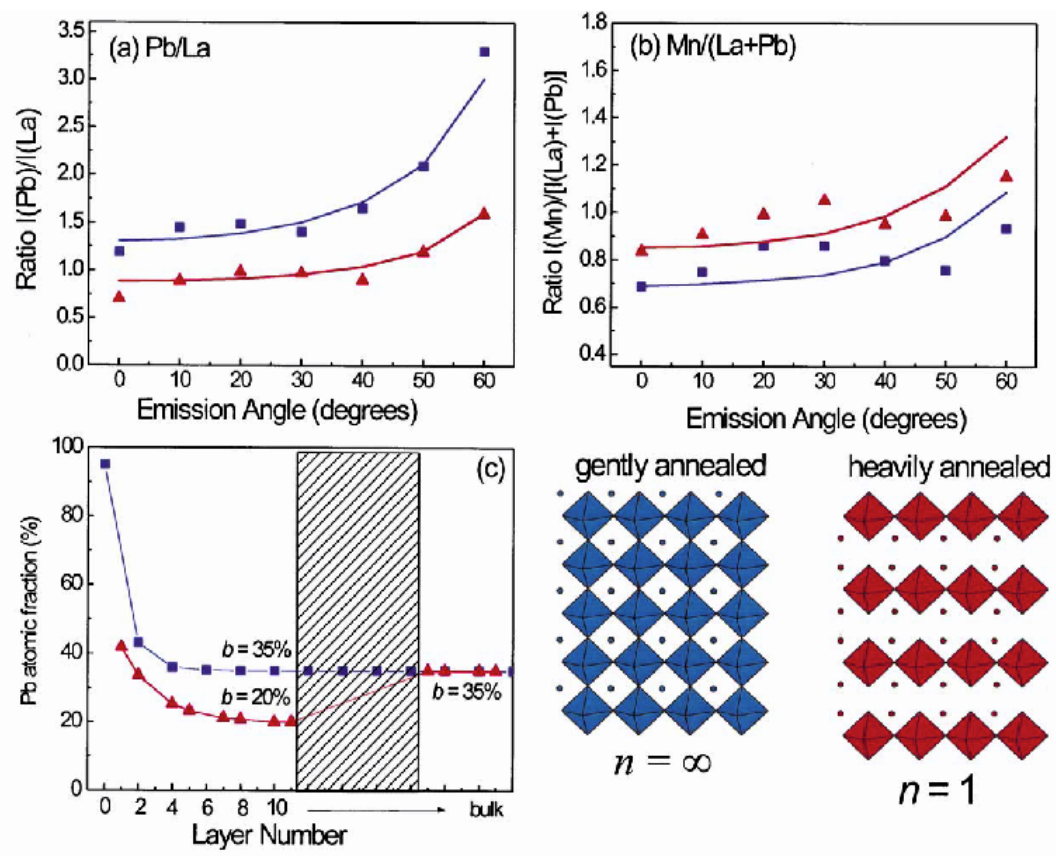

Figure 1. Panel (a) shows the normalized relative XPS core level intensity ratios of $\mathrm{Pb}(4 f)$ peaks to that of $\mathrm{La}(3 d)$ peaks and (b) shows the corresponding experimental and calculated intensity ratios of $\mathrm{Mn}$ to $(\mathrm{La}+\mathrm{Pb})$ core level intensities. The best fits (-) for the gently annealed surface $(\boldsymbol{\square})$ and for the heavily annealed surface $(\boldsymbol{\Delta})$ of $\mathrm{La}_{0.65} \mathrm{~Pb}_{0.35} \mathrm{MnO}_{3}$, as a function of photoelectron emission angle $(\theta)$, are compared with the data. Panel (c) displays the resulting calculated $\mathrm{Pb}$ atomic fractions as a function of layer number. The inset shows schematically the layers stacking sequence used to obtain the compositional fits in panels (a) and (b) for the gently (left) and heavily (right) annealed surfaces. Adapted from [13]. 
There is a major problem that the usual means for estimating electron mean free paths in solids are not readily applicable to free and isolated clusters, particularly small clusters. As an example of the complexities of estimating electron mean free path in clusters, Auger electron emission from argon clusters are seen to be more surface sensitive than photoelectrons of a similar kinetic energy [34] and this cannot be easily related the number of valence electrons nor the plasmons.

There are examples of segregation in small free clusters, as described below, in spite of the difficulties in characterization surface composition of a very small free cluster, or cluster assembled material. Some studies of surface segregation, in clusters, have tended exploited the small difference in binding energy for atoms place at the surface compared to the bulk (the surface to bulk core level shift [35]). For cluster assembled materials, such a signature must consider the boundary between clusters as well as the surface. So this latter technique must be applied with care.

\section{THE ENERGETICS OF SEGREGATION}

Over the past many decades, the phenomenon of surface segregation attracted considerable theoretical interest. Various theories have been developed in order to account for the enrichment at the binary alloy surface. First-principles approach [36], embeddedatom method (EAM) model [37], Finnis-Sinclair (FS) potential [38], and BozzoloFerrante-Smith (BFS) method [39] have all been used to simulate the surface segregation for fcc type random and ordered alloys, while for fcc and hcp lattices, the modified analytic embedded-atom method (MAEAM) many-body potential is used to theoretically study the surface segregation phenomena of binary alloys [40]. Unfortunately, there are no detailed finite temperature theoretical studies of the surface segregation of multicomponent half-metal alloys, and as yet, little has been done to model non-equilibrium segregation in multi-component systems. Monte Carlo simulations have provided some insight and have more extensively employed to model segregation in clusters. It is a challenging vista of opportunity for both the theorist and experimentalist.

During the early 1980's on binary alloys, Moran-Lopez [41] found that they could predict between two metals, which would act as the solvent and which as a solute. Of the many binary systems they considered, it was only those which contained transition metal elements, which were known to order magnetically (ferromagnetic, anti-ferromagnetic, etc.) that deviated from expectation. Magnetic ordering, not often well understood at the surface of a cluster, must therefore be considered as well. Ternary systems containing components with magnetic moments are even more complex. For example, for two very similar ternary semi-Heusler alloys, NiMnSb and $\mathrm{TiCoSb}$, the resultant segregation could not be predicted from classical mechanism arguments [14,42]. In the case of NiMnSb, it was found that Mn enriched the surface layers whereas for TiCoSb, Co and Sb dominated the surface and sub-surface regions, respectively.

In considering the influence of magnetic moments upon segregation, one must go back to basic ideas to identify what magnetic ordering would occur and justify why such ordering is energetically favorable. For $\mathrm{Cr}$ and $\mathrm{Mn}$ alone in bulk or thin layers, their spin coupling leads to an anti-ferromagnetic alignment; although, depending on crystal orientation, uncompensated spins can exist at the surface or buried interface. However, if $\mathrm{Cr}$ or $\mathrm{Mn}$ is alloyed with another element, ferromagnetic ordering is very possible, for 
example in $\mathrm{BiMnO}_{3}$ and $\mathrm{CrO}_{2}$. There is also the need to consider the effective Curie temperature, as with decreases in the ferromagnetic layer thickness there should be decreases in $T_{C}$ (finite size scaling). These subtle possible contributions to the surface energy due to magnetic ordering are illustrated schematically in Figure 2.

As we have already noted, in order to minimize the free energy difference, surface segregation, surface reconstruction or/and surface relaxation compete, making most efforts to estimate the energetics of segregation in multicomponent systems, with more than two components, quite complex. Given that the presence of surface segregation is an indicator of a difference in free energy (chemical potential) between the surface and the

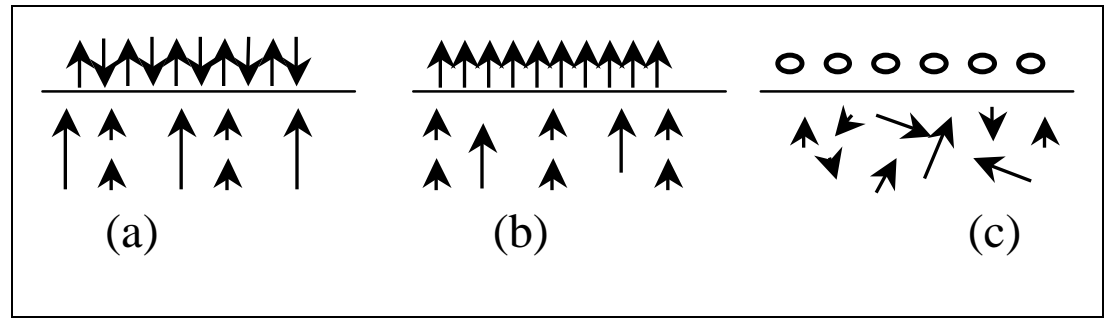

Figure 2. Surface ordering of moments in (a) anti-ferromagnetic ordering (b) ferromagnetic ordering (c) non-magnetic insulator models which could provide lowering of difference in surface free energy. Adapted from [42].

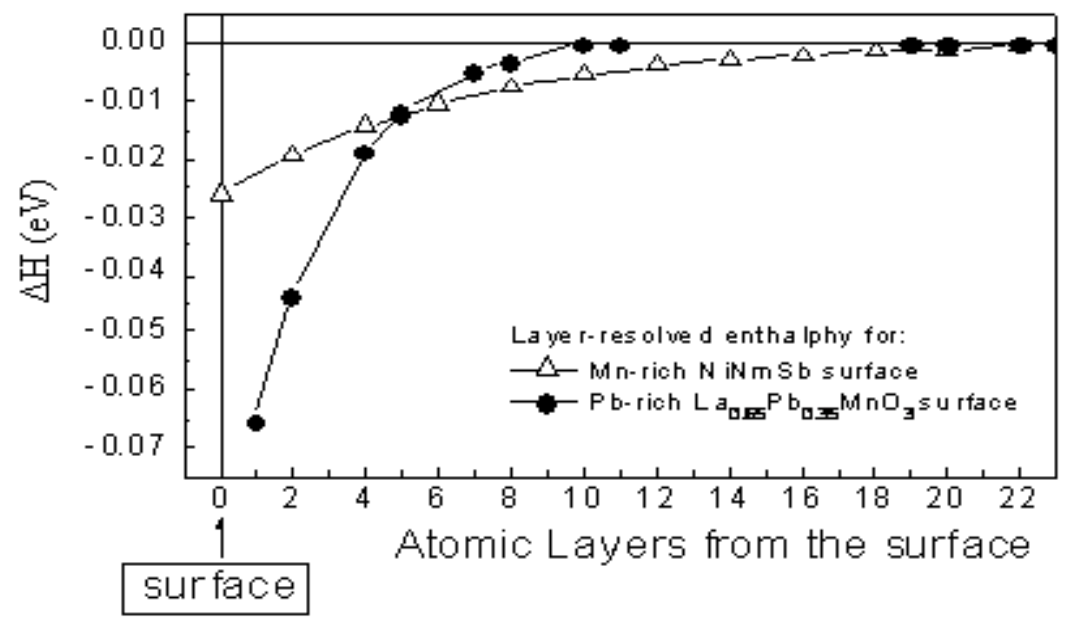

Figure 3. The enthalpy difference $(\Delta \mathrm{H})$ between the surface and the bulk based on segregations studies of $\mathrm{NiMnSb}[9,14]$ and $\mathrm{La}_{0.65} \mathrm{~Pb}_{0.35} \mathrm{MnO}_{3}$ [13].

bulk [1], estimates of the differences in the surface enthalpy relative to the bulk, based upon experiment can be quite valuable [2]. This energy difference is typically calculated from the experimentally determined segregation profiles using the approximation:

$f_{j}(A) / f_{j}(B)=\left[f_{b}(A) / f_{b}(B)\right] \exp \left(-\Delta H / k_{B} T\right)$ 
where $f_{b}(A)$ and $f_{b}(B)$ are the bulk layer concentrations of constituents $A$ and $B$, and $T$ is the annealing temperature [43]. These enthalpy differences exists between the surface and the bulk have been estimated for the half Heusler alloy NiMnSb(100) surface $[9,14,42]$, based on most the extensive $\mathrm{Mn}$ segregated surface of $\mathrm{NiMnSb}$, as well as for the $\mathrm{Pb}$ segregated surface of $\mathrm{La}_{0.65} \mathrm{~Pb}_{0.35} \mathrm{MnO}_{3}$ [13], as indicated in Figure 3, among other systems studied $[1,2,10]$.

Any effort to extend this type of analysis to clusters is simplified by the small volumes, but when the cluster is too large to be amenable to a molecular calculation, simplifications are typically applied: shallow $4 \mathrm{f}$ or $5 \mathrm{~d}$ levels are treated as core levels, and neglect of spin-orbit coupling, magnetic ordering energies, correlation energies, lattice relations are among the simplifications commonly undertaken. All of this tends to make us suspect that segregation in clusters is often more rapid and more extensive than one gets from an inspection of much of the cluster assembled materials literature.

\section{SEGREGATION IN WEAKLY COHESIVE CLUSTERS}

It is not necessary for there to be a strong chemical potential for surface segregation to occur in a cluster. A good example is the van der Waals "like" clusters. Radial surface segregation has been demonstrated in the self-assembled $\mathrm{Ar} / \mathrm{Xe}$ and $\mathrm{Ar} / \mathrm{Kr}$ clusters formed in an adiabatic expansion $[15,16]$. In both types of free gas clusters, there is a clear preference for the lower cohesive energy atoms to occupy the surface and leave higher cohesive energy atoms in the bulk. As a first approximation, the surface energy is proportional to the faction of missing bonds of the surface atoms and the cohesive energy. Consequently, quasi-spherical shapes and placing lower cohesive energy on the surface can minimize the surface cost.

Because of the much higher condensation temperature for Xe compared to Ar, little $\mathrm{Xe}$ is needed in the primary gas mixture to make the resulting free clusters, from gas expansion, mostly Xe. When the clusters are composed of both $\mathrm{Xe}$ and $\mathrm{Ar}$ atoms (or $\mathrm{Kr}$ and $\mathrm{Ar}$ atoms), the $\mathrm{Ar} 2 \mathrm{p}$ spectra tends to have a dominant signature of argon occupying surface sites. In Figure 4, the XPS spectra of the Ar 2p and Xe 4d core levels show that argon segregates to surface the Ar/Xe clusters from the proportionally greater surface contribution to the $2 \mathrm{p}$ core level [16]. For the higher Xe/Ar ratio (5.3\% primary gas mixing contribution) dominant peaks in $\mathrm{Ar} 2 \mathrm{p}$ spectra $\mathrm{I}_{\mathrm{Ar}}$ and $\mathrm{S}_{\mathrm{Ar}}$ indicate that both $\mathrm{Ar}$ and Xe atoms both occupies the surface, corresponding to the high surface peak in Xe spectra. For a primary gas mixing ratio of $3.2 \% \mathrm{Xe}$, besides Ar-Xe mixed surface, increasing intensity of $\mathrm{I}_{\mathrm{Xe}}$ and decreasing intensity of the surface Xe contribution suggests that a Xe interface layer is formed between the Xe bulk and Ar surface. With the increase of Ar, the mixture of Ar and Xe at the surface is replaced by almost all Ar [16].

Mixed Ar/Kr clusters exhibit similar radial surface segregation of Ar atoms at the surface while $\mathrm{Kr}$ atoms tend to dominate the bulk [15]. No interface layer has been observed in these mixed $\mathrm{Kr}$ and Ar clusters due to the relatively small difference of cohesive energy between $\mathrm{Ar}$ and $\mathrm{Kr}$ [15]. Resonant excitation processes, such as occurs in resonant photoemission, and near edge X-ray absorption fine structure spectroscopy, can significantly increase surface sensitivity. The shifts between the surface and bulk contributions, even in pure argon clusters are readily identified [44]. 


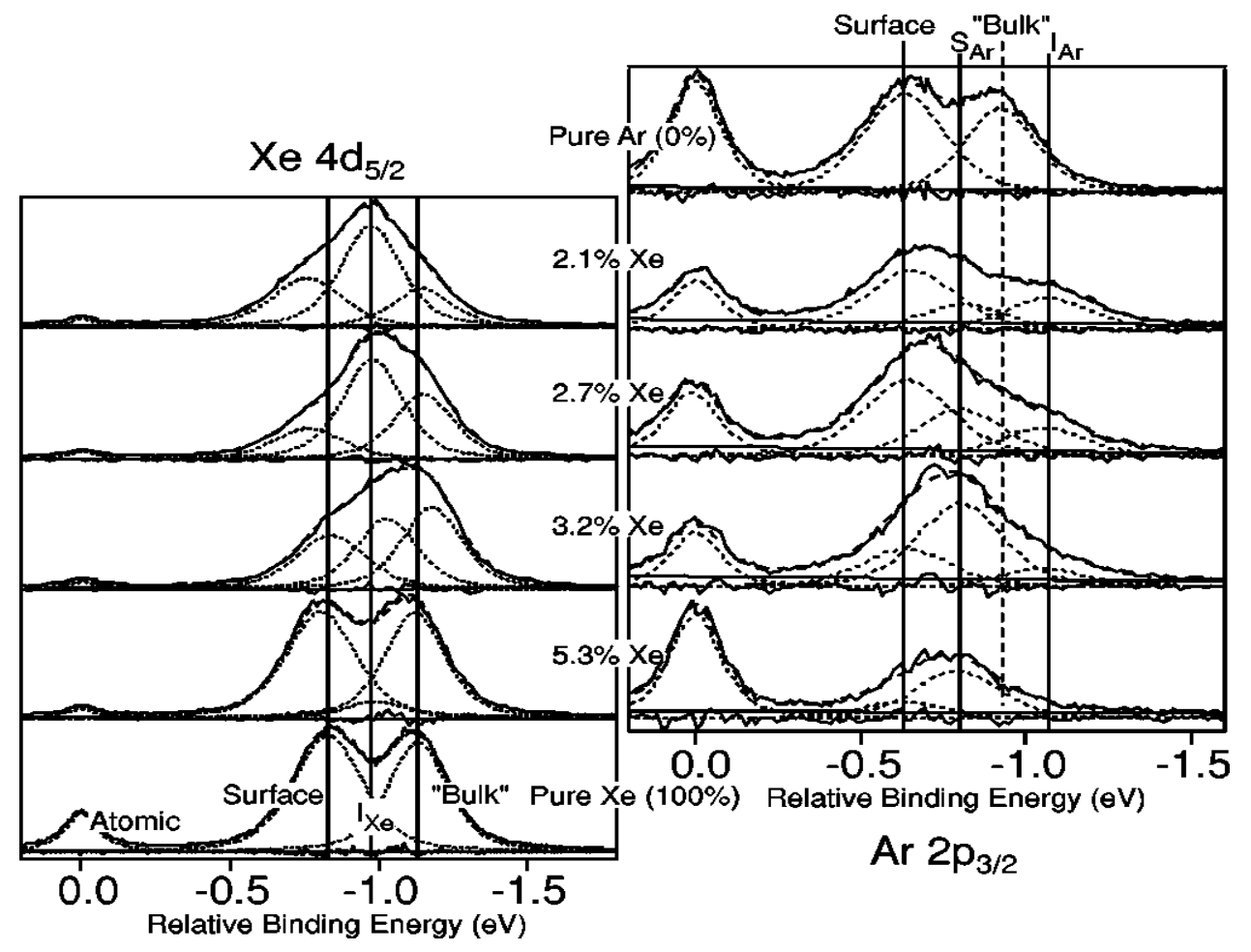

Figure 4. The XPS spectra of the $\operatorname{Ar} 2 p_{3 / 2}$ and Xe $4 d_{5 / 2}$ core levels for clusters produced from different primary gas mixing ratios. The peak at zero relative binding energy corresponds to the atomic peak. Adapted from [16].

\section{SEGREGATION IN CLUSTERS WITH SHORT RANGE ORDER}

Surface segregation is a known phenomenon in mixed component clusters, which can be treated as solid solution, but short range order can be extremely persistent in many alloy materials, causing notable deviations from an ideal solution. So is the strong ordering seen in bulk alloys observed at the surfaces of small isolated clusters of the same composition? Generally this is not likely to occur.

FePt nanoparticles are considered as a promising candidate material for ultrahigh density magnetic storage media, because of the large magnetic anisotropy energies. Monte Carlo simulations have been applied on this binary metallic cluster system in the $\mathrm{L}_{0}$ phase $[45,46]$. Significant segregation is indicated if the difference of surface energy between pure elements $\mathrm{Fe}$ and $\mathrm{Pt}$ is considered [45]. This energy difference results in an energetic driving force for surface segregation. Such segregation will alter with temperature and cluster size, but can alter an ordered cluster from one with large nearest neighbor short range order to a cluster with a strong preference for surface enrichment by $\mathrm{Pt}$ (white) atoms [45], as indicated in Figure 5. A reduction of nanoparticles size can decrease ordering temperature, so that at as the cluster size decreases, disorder increases for the same finite temperature [46]. Because of the enhancement of surface to volume 
ratio with decreasing particle size, it is again preferential to place low surface energy atoms in the surface.
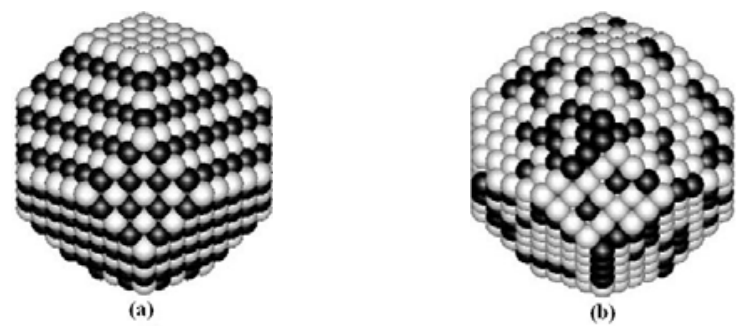

Figure 5. A comparison of (a) an ordered $3.3 \mathrm{~nm}$ FePt nanoparticle, with (b) an equilibrium cluster above the ordering temperature. White is Pt and dark is Fe. Adapted from [45].

\section{SEGREGATION IN BaFe ${ }_{12-2 \mathrm{x}} \mathrm{Co}_{\mathrm{x}} \mathrm{Ti}_{\mathrm{x}} \mathrm{O}_{19}$ CLUSTER ASSEMBLED MATERIALS}

Surface segregation can also be found in $\mathrm{BaFe}_{12-2 \mathrm{x}} \mathrm{Co}_{\mathrm{x}} \mathrm{Ti}_{\mathrm{x}} \mathrm{O}_{19}$ barium ferrite nanoparticles [47]. Resonant photoemission results show that cobalt and titanium dopants strongly hybridize with the barium ferrite matrix. Due to the strong resonant behavior, we can find different element contributions to the various occupied valence bands in the system. It was found that the enhancement of resonance in $1.8 \mathrm{eV}$ valence band can result from both barium and cobalt contributions. Extensive annealing (at $685 \mathrm{~K}$ for 10 hours in vacuum) of the sample leads to enhancement of the feature at $1.8 \mathrm{eV}$ binding energy, indicating that barium and cobalt are enriched near the surface region, while iron is depleted at the surface compared to the bulk $\mathrm{BaFe}_{12-2 \mathrm{x}} \mathrm{Co}_{\mathrm{x}} \mathrm{Ti}_{\mathrm{x}} \mathrm{O}_{19}$. This segregation is evident from the shift in the spectral peaks near $60 \mathrm{eV}$ from the broad feature at $61 \mathrm{eV}$ (unoxidized Co $3 \mathrm{p}$ is nominally at $58.9 \mathrm{eV}$ binding energy, while unoxidized $\mathrm{Ti} 3 \mathrm{~s}$ is nominally at $58.7 \mathrm{eV}$ ) to features at $58 \mathrm{eV}$ and $56 \mathrm{eV}$ binding energy (unoxidized $\mathrm{Fe} 3 \mathrm{p}$ is nominally at $52.7 \mathrm{eV}$ binding energy). Figure 6 shows some of the evidence of the surface segregation phenomenon in this system. Such Co oxide segregation, following extensive annealing, does not require a magnetically dead layer at the surface of the crystallites as ferromagnetic Co doped titanium oxide is known.

While there has been some speculation concerning a dead layer in cobalt and titanium doped barium ferrites, from magnetic modeling [48], such a layer would be less than $1 \mathrm{~nm}$ to be consistent with the photoemission results. The limited mean free path of the photoelectrons indicates that the segregation layer is quite thin, even after extensive annealing, consistent with the very short argon ion sputtering time needed to remove the segregation layer. Even if the segregation leads to a material that is nominally paramagnetic, solutions to the Landau-Ginzburg equation [49] indicate that a layer so thin $(<1 \mathrm{~nm})$ would have an induced magnetization by proximity.

In this system the very large number of components, with competing contributions to the electronic structure, lattice strain, and the availability of a large number of different oxidation states, so magnetic ordering may not even play a dominant role in the surface segregation, but it cannot be neglected $a$ priori. 


\section{SUMMARY}

Surface segregation in alloy free clusters as well as surface and grain boundary segregation in cluster assembled materials must be taken as a given, unless there is sufficient evidence to indicate otherwise. Strong alloy ordering does not necessarily impart compositional stability to the surface, nor does weak inter-atomic interactions. Even at very low finite temperatures segregation may still occur, particularly if there is a high density of lattice vibrations, so segregation can occur even for "refractory" alloy clusters. Rigid refractory solids are not immune from surface segregation, with surface segregation evident at temperatures that are sometimes just a very small fraction of the melting temperature (2-3\%). The tendency for surface segregation to occur in clusters may be exacerbated.
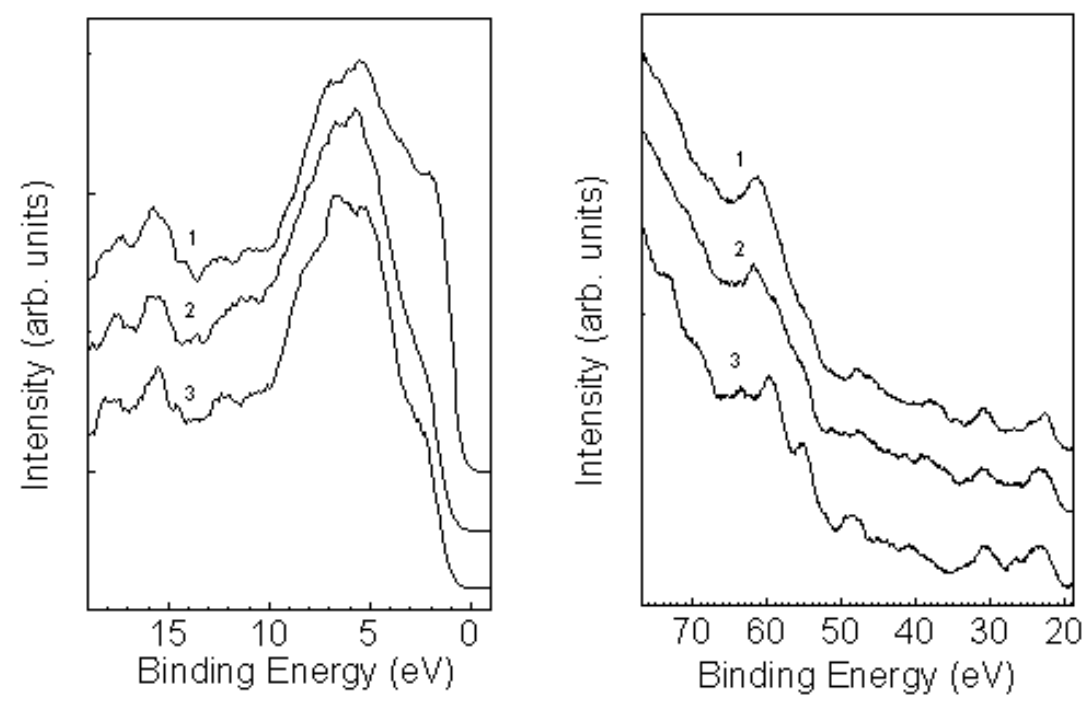

Figure 6. Photoemission spectra taken at room temperature after prolonged heating followed after several short heating/sputtering cycles: (1) prolonged annealing at $685 \mathrm{~K}$, (2) after brief heating, (3) after sputtering. Spectra of the Co 3p and Fe 3p shallow cores (right panel), shows enhancement of $\mathrm{Co} 3 \mathrm{p}$ and/or Co and Ba Auger with annealing while the enhancement of the valence band $1.8 \mathrm{eV}$ binding energy feature is shown at left. The photon energy was $115 \mathrm{eV}$. Adapted from [47].

\section{ACKNOWLEDGEMENTS}

The support of the Center for Advanced Microstructures and Devices (CAMD) and the Louisiana Board of Regents, the NSF "QSPINS" MRSEC (DMR 0213808), and the financial support by the DFG within the SPP1104 (contract no. Mo940/1) are gratefully acknowledged. We also acknowledged useful conversations with Olle Bjørnehom, Eckart Rühl and the contributions of C. Borca, A.N. Caruso and H.J. Jeong to the work. 


\section{References}

[1] P.A. Dowben and A. Miller, editors, Surface Segregation Phenomena, (CRC Press, Boston, 1990) p. 145.

[2] P.A. Dowben and S.J. Jenkins, in Frontiers in Magnetic Materials, edited by Anant Narlikar (Springer Verlag, 2005), pp. 295-325.

[3] D. Ristoiu, J. P. Nozières, C. N. Borca, T. Komesu, H. -K. Jeong and P. A. Dowben, Europhys. Lett. 49, 624 (2000).

[4] G. L. Bona, F. Meier and M. Taborelli, Solid State Commun. 56, 391 (1985).

[5] W. Zhu, B. Sinkovic, E. Vescovo, C. Tanaka and J. S. Moodera, Phys. Rev. B 64, 060403 (2001).

[6] C.N. Borca, B. Xu, T. Komesu, H.-K. Jeong, M.T. Liu, S.-H. Liou, S. Stadler, Y. Idzerda and P. A. Dowben, Europhys. Lett. 56, 722 (2001).

[7] L. Ritchie, G. Xiao, Y. Ji, T. Y. Chen, C. L. Chien, M. Zhang, J. Chen, Z. Liu, G. Wu and X.X. Zhang, Phys. Rev. B 68, 104430 (2003).

[8] A. Plecenik, K. Fröhlich, J. P. Espinós, J. P. Holgado, A. Halabica, M. Pripko and A. Gilabert, Appl. Phys. Lett. 81, 859 (2002).

[9] D. Ristoiu, J. P. Nozières, C. N. Broca, B. Borca and P.A. Dowben, Appl. Phys. Lett. 76, 2349 (2000).

[10] J. Choi, J. Zhang, S.-H. Liou, P.A. Dowben and E.W. Plummer, Phys. Rev. B 59, 13453 (1999).

[11] H. Dulli, P.A. Dowben, S.-H. Liou, E.W. Plummer, Phys. Rev. B 62, R14629 (2000).

[12] H. Dulli, E.W. Plummer, P.A. Dowben, J. Choi and S.-H. Liou, Appl. Phys. Lett. 77, 570-572 (2000).

[13] C.N. Borca, Bo Xu, T. Komesu, H.-K. Jeong, M.T. Liu, S.-H. Liou, P.A. Dowben, Surface Science Letters 512, L346 (2002).

[14] A.N. Caruso, C.N. Borca, D. Ristoiu, J.P. Nozières, P.A. Dowben, Surf. Sci. 525, L109-L115 (2003).

[15] M. Tchaplyguine, M. Lundwall, M. Gisselbrecht, G. Öhrwall, R. Feifel, S. Sorensen, S. Svensson, N. Mårtensson, O. Björneholm, Phys. Rev. A 69, 031201 (2004).

[16] M. Lundwall, M. Tchaplyguine, G. Öhrwall, R. Feifel, A. Lindblad, A. Lindgren, S. Sorensen, S. Svensson, O. Björneholm, Chem. Phys. Lett. 392, 433-438 (2004).

[17] H.W. Werner and R P H Garten, Rep. Prog. Phys. 47, 221 (1984).

[18] S. Hofmann, Surf. Interface Anal. 9, 3 (1986).

[19] D. Briggs and M.P. Seah, editors, Practical Surface Analysis by XPS and AES, (Wiley, Chichester, 1983).

[20] W. L. Baun, Surf. Interface Anal. 3, 243 (1981).

[21] S. A. Benninghoven, F. G. Rudenauer and H. W. Werner, Secondary Ion Mass Spectrometry, (Wiley, New York, 1987).

[22] H.-K. Jeong, T. Komesu, C.-S. Yang, P. A. Dowben, B. D. Schultz and C. J. Palmstrøm, Mater. Lett. 58, 2993 (2004).

[23] M.P. Seah, M.E. Jones and M.T. Anthony, Surf. Interface Anal. 6, 242 (1984).

[24] M.P. Seah, Surf. Interface Anal. 20, 243 (1993).

[25] S. Tanuma, S. Ichimura, and K. Yoshihara, Appl. Surf. Sci. 101, 47 (1996). 
[26] NIST Electron Inelastic Mean Free Path program, ver. 1.1 (NST std. ref. Database 71) http://www.nist.gov.srd

[27] P.A. Dowben, M. Grunze and D. Wright, Surf. Sci. 134, L524-L582 (1983).

[28] U. Vahalia, P.A. Dowben and A. Miller, J. Electron Spectrosc. Rel. Phenom. 37, 303 (1986).

[29] S. Tamura, C.J. Powell, and D.R. Penn, Surf. Interface Anal. 17, 911 (1991).

[30] S. Tamura, C.J. Powell, and D.R. Penn, Surf. Interface Anal. 21, 165 (1994).

[31] C.J. Powell and A. Jablonski, J. Vac. Sci. Technol. A17, 1122 (1999).

[32] G. Schönhense and H.C. Siegmann, Ann. Phys. (Leipzig) 2, 465 (1993).

[33] H.-J. Drouhin, Phys. Rev. B 56, 14886 (1997).

[34] M. Lundwall, M. Tchaplyguine, G. Öhrwall, A. Lindblad, S. Peredkov, T. Rander, S. Svensson, and O. Bjørneholm, Surface Sci. (2005) in press

[35] D. Spanjaard, C. Guillot, M.-C. Desjonqueres, G. Treglia, and J. Lecante, Surf. Sci. Repts. 5, 1 (1985).

[36] R. Monnier, Phil. Mag. B 75, 67-144 (1997).

[37] S.M. Foiles, Phys. Rev. B 32, 7685 (1985).

[38] M.W. Finnis and J.E. Sinclair, Phil. Mag. A50, 45 (1984).

[39] G. Bozzolo, J. Ferrante, R.D. Noebe, B. Good, F.S. Honecy and P. Abel, Comput. Mater. Sci. 15, 169 (1999).

[40] H. Deng, W. Hu, X. Shu and B. Zhang, Appl. Surf. Sci. 221, 408 (2004).

[41] J.L. Moran-Lopez, Surf. Sci. Lett. 188, L472 (1987).

[42] A.N. Caruso and C.N. Borca, in Recent Developments in Magnetism and Magnetic Materials 1, pp. 33-48 (2003).

[43] S. Y. Liu and H. H. Kung, Surf. Sci. 110, 504 (1981).

[44] A. Knop, D.N. McIlroy, P.A. Dowben, and E. Rühl, in Two-Center Effects in IonAtom Collisions, T. J. Gay and A.F. Starace editors, (AIP Conference Proceedings 362, 1996) pp. 274-280.

[45] B.Yang, M.Asta, O.N. Mryasov, T.J. Klemmer and R.W. Chantrell, Scripta Materialia 53, 417-422 (2005).

[46] R.V. Chepulskii, J. Velev and W.H. Bulter, J. Appl. Phys. 97, 10J311 (2005).

[47] Natalie Palina, H, Modrow, R. Müller, J. Hormes, P.A. Dowben and Ya.B. Losovyj, Materials Letter, (2005) in press.

[48] S. Kurisu, T. Ido and H. Yokoyama, IEEE Trans. Magn. MAG-23, 3137 (1987).

[49] A. Miller and P.A. Dowben, J. Phys. Cond. Matt. 5, 5459 (1993). 